\begin{tabular}{|c|c|c|}
\hline \multirow{3}{*}{$\begin{array}{l}\text { EREM 75/2 } \\
\text { Journal of Environmental Research, } \\
\text { Engineering and Management } \\
\text { Vol. } 75 \text { / No. } 2 \text { / } 2019 \\
\text { pp. } 28-35 \\
\text { Dol 10.5755/j01.erem.75.2.22352 }\end{array}$} & \multicolumn{2}{|c|}{$\begin{array}{l}\text { A New Method of Determining the State of } \\
\text { Water and Agricultural Areas in Real Time }\end{array}$} \\
\hline & Received 2018/12 & Accepted after revision 2019/03 \\
\hline & \multicolumn{2}{|c|}{ crossef http://dx.doi.org/10.5755/j01.erem.75.2.22352 } \\
\hline
\end{tabular}

\title{
A New Method of Determining the State of Water and Agricultural Areas in Real Time
}

\section{N. S. Myazin*}

Peter the Great Saint Petersburg Polytechnical University, Russia, Saint Petersburg, 195251, 29 Polytechniheskya str.

\section{V. Davydov}

Peter the Great Saint Petersburg Polytechnical University, Russia, Saint Petersburg, 195251, 29 Polytechniheskya str. All Russian Research Institute of Phytopathology, Russia, Moscow Region, 143050, Moscow Region, Odintsovo district, B. Vyazyomy, Institute str., ownership 5

\section{V. Yushkova}

Saint Petersburg University of Management Technologies and Economics

Russia, Saint Petersburg 190103, 44, Lermontovsky prospect

\section{Yu. Rud'}

All Russian Research Institute of Phytopathology, Russia, Moscow Region, 143050, Moscow Region, Odintsovo district, B. Vyazyomy, Institute str., ownership 5

\section{${ }^{*}$ Corresponding authors: myazin.n@list.ru}

The paper discusses the technique of environmental condition monitoring based on the phenomenon of nuclear magnetic resonance (NMR). Express control is an integral part of agriculture. It is especially necessary when working on the farm or in the fields. In this case, situations often arise when it is impossible to deliver samples for research in a stationary laboratory (e.g., results are needed immediately or samples can change their properties during transportation). Now, there are many methods for monitoring the states of media in the express mode. Therefore, a brief review of these methods as well as their limitations was carried out. To overcome these limitations, a new technological solution was proposed for the creation of an NMR spectrometer. 
The proposed compact NMR spectrometer allows measuring the longitudinal and transverse relaxation times of the medium. To enhance its functionality, a new magnetic system and a signal registration circuit have been developed. These improvements allow detection of the NMR signal at different frequencies, thus registering the signal spectrum. With the help of the proposed device, studies of various media have been carried out; the results of these studies are presented.

Keywords: agricultural grounds, ecological monitoring, nuclear magnetic resonance, magnetic field, spectroscopy.

\section{Introduction}

In conditions of population growth and reduction of acreage, the role of efficient and rational use of agricultural lands is growing. Environmental monitoring is one of the key elements to achieve this (Arkhipov, 2017; Davydov et al., 2014; Davydov et al., 2018; Karseev et al., 2015). New tasks that arise in the process of environmental monitoring require research in the express mode, based on the results of which it would be possible to take a decision both on the state of the environment and on its further use. This is especially important during water environment testing (Arkhipov, 2017; Davydov and Myazin, 2017a; Davydov et al., 2017b; Karseev et al., 2015). For such researches of condensed media in the express mode, a large number of methods and instruments have been developed for environmental monitoring using various physical phenomena (Arkhipov, 2017; Bochenin, 2007; Davydov et al., 2017c; Karseev et al., 2015).

At present, the most used methods for express control of various media are the ones that do not significantly change the physical structure and chemical composition of the medium. This is because workers have to investigate in details the sample of the medium in a stationary laboratory if they detect deviation in the state of it during express analysis (Davydov et al., 2014; Davydov and Myazin, 2017a; Davydov et al., 2017b; Vitkovskii and Marusina, 2016). Such methods include optical and nuclear magnetic resonance methods.

Devices for express control, developed on the basis of optical methods, are mainly used to control the state of a small number of media. For example, express analyser IT-1 for determination of the water percentage in engine or transformer oil and diesel fuel, the portable photometer pHotoFlex (for determination of the water condition and $\mathrm{pH}$ ), the visible light portable spectrophotometer DR 190 manufactured by $\mathrm{HACH}$ LANGE for the liquid media condition control with the turbidity not more than $10 \mathrm{FNU}$, etc. Their use for environmental monitoring, especially when carrying out work in hard-to-reach places like forest tracts, agricultural lands, water bodies and their coastal zones, where different chemical elements can be found in the studied media, is not appropriate.

Devices based on the phenomenon of nuclear magnetic resonance (NMR) surpass in their functional capabilities the optical methods at least by an order of magnitude. In the previously developed NMR design of the relaxometer (Davydov et al., 2014; Davydov and Myazin, 2017a; Karseev et al., 2015), the express control consisted of recording the NMR signal from various liquid media at the resonant frequency of the protons. After this, using NMR signals, we can determine the times of longitudinal and transverse relaxation of the medium $T_{1}$ and $T_{2}$. Comparing them with the basic values, corresponding to the standard state of the investigated medium, the presence of impurities in it was instantaneously determined.

However, experience with the operation of a smallsized NMR relaxometer has shown that during environmental monitoring this may not be enough to make a decision at the measurement site for further use of the investigated medium. The conducted experiments have shown that the aqueous media, depending on the natural conditions, might contain chemical elements (e.g., fluorine, potassium, magnesium, phosphorus, etc.) that are harmless in small quantities for living organisms. Their effect on the shape of the NMR signal is difficult to predict (Davydov et al., 2017b; Myazin et al., 2017); these elements cause 
significant changes in $T_{1}$ and $T_{2}$. For this reason, after measuring $T_{1}$ and $T_{2}$, it may be concluded that the researched medium represents a potential hazard. Thus, it is possible to make wrong decisions, which leads to additional spending on researching samples of this medium.

Therefore, it is important not only to detect a change in the investigated medium, but also to establish its cause on the site of sampling. This in some cases allows you to take on-site measures to eliminate the problem. For example, potassium nitrate is diluted with water before use. During the test, it turned out that the potassium concentration was too high in the prepared aqueous solution. The NMR test in the express mode showed that the concentration of potassium in the saltpetre was above the norm. Recalculation of the ratio of water and saltpetre in the produced solution showed that we should add more water. After this, this solution was ready for use.

Other chemical elements, such as fluorine, magnesium, phosphorus, are used in fertilisers, sodium and chlorine in technical fluids for various purposes. These chemical elements come from fields or technical agricultural zones into water bodies and cause changes in the aquatic environment.

The nuclei of the marked chemical elements have a magnetic moment, which makes it possible to detect the NMR signal in a magnetic field at their resonance frequencies. The ratio of the amplitudes of the detected NMR signals determines the relative concentration of these chemical elements in the medium. The implementation of the device that makes it possible to carry out such measurements is the purpose of this paper.

\section{Methods}

Since the device should be able to perform measurements in real time in various situations (including measurements in the field), it must be light enough to allow transportation from one place to another. Unfortunately, that feature is not available for the NMR devices based on a standard impulse technique. This is because measurements using the impulse technique require a powerful power supply that cannot be provided in the field. Moreover, the device cannot have large magnets with a strong magnetic field, since they negatively affect the weight of the device. Therefore, all measurements should be performed in a weak magnetic field.

To create a device that meets the specified mobility requirements, it was proposed to use a modulation technique. The basic idea of the modulation technique is as follows. The investigated medium is placed in a weak magnetic field that consists of two fields. The first field is a constant magnetic field from a permanent magnet. The second field is an alternating magnetic field from the modulation coil. In that case, the total value of magnetic field varies as follows:

$$
H=H_{0}+H_{m} \sin \left(\omega_{m} t\right)
$$

where $H_{0}$ - constant magnetic field, $H_{m}$ - modulation coil field, $\omega_{m}$ - modulation frequency.

Knowing this, it is possible to register the NMR signal from a medium at a medium resonant frequency, which is defined as follows:

$$
\omega=\gamma H=\gamma H_{0}+\gamma H_{m} \sin \left(\omega_{m} t\right),
$$

where $y$ is a gyromagnetic ratio of a nuclei.

Thus, the registration of the NMR signal is possible at any frequency defined by equation 2 . However, one of the features of registering the NMR signal in a weak magnetic field using the modulation technique is that its registering should be performed only at the resonance frequency $\left(\omega=\omega_{0}=\gamma H_{0}\right)$ (Davydov et al., 2014). That frequency corresponds to the maximum of a signal-to-noise ratio.

Finally, since different atomic nuclei have different gyromagnetic ratios, it is possible to register the NMR signal from different nuclei on different frequencies, thus registering a medium spectrum.

However, it should be noted that the sensitivity of various nuclei to the NMR method might vary widely. The difference in sensitivity can be up to four orders of magnitude. In a rough approximation, the signal-tonoise ratio is proportional to the sensitivity. Therefore, in a weak magnetic field, it is possible to register the NMR signal only from the nuclei that have sensitivity comparable with proton sensitivity (protons have 
the highest sensitivity). This is a strong limitation on a possible usage of a modulation technique in a weak magnetic field during environmental monitoring. Yet in practice, it is often enough to receive a signal from those nuclei that are sensitive enough to NMR methods (e.g., fluorine, phosphorus, boron, and sodium).

\section{Compact NMR Spectrometer}

One of the main tasks that has been developed is a new design of a compact laboratory nuclear magnetic spectrometer that allows (in contrast to NMR relaxometers) detecting NMR signals from different nuclei with magnetic moments contained in the sample of the medium under study. A compact NMR spectrometer should have high accuracy characteristics (measurement error less than 1\%). Moreover, it should be easy to transport and have autonomous supply (batteries). The results of the measurements performed by this device, after conversion, must be sent to the input of the laptop, which processes them and gives the final information about the state of the investigated medium.

Fig. 1 shows the block diagram of a compact NMR spectrometer with the new construction elements that have been developed.

The NMR spectrometer consists of a permanent magnet (1), special inserts (2), neutral for the placement and alignment of the magnets (3), adjusting screws (4), modulation coil (5), NMR signal registration coil

Fig. 1

The block diagram of a compact NMR spectrometer

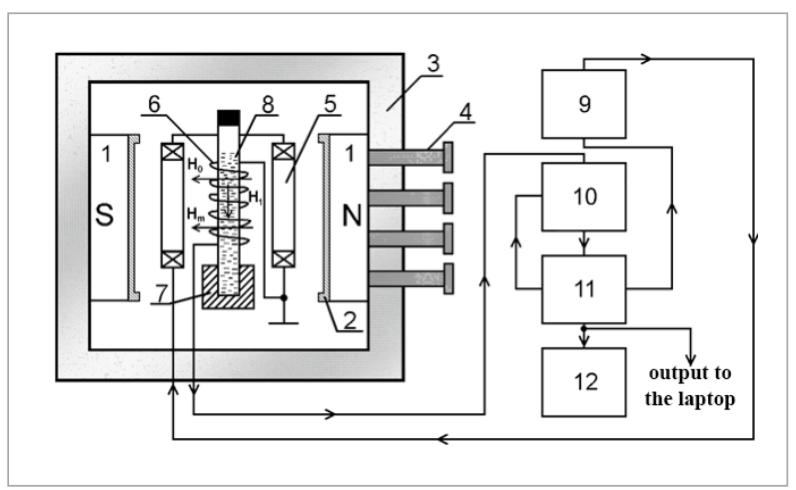

(6), locking device for the container with the researched medium (7), container with the researched medium (8), magnetic field modulation generator (9), registration scheme including RF autodyne generator (10), processing and control unit (11) and an oscilloscope (12) that is needed for tuning the device.

The small-sized magnetic system was made of the material NdFeB in the form of a disk 1 with a large residual induction. This allowed at the diameter of the magnet 1 poles $d_{m}=92 \mathrm{~mm}$ and distance between them $d_{z}=16 \mathrm{~mm}$ to provide in the area of the registration coil 6 the inhomogeneity $0.5 \cdot 10^{3} \mathrm{~cm}^{1}$ at the induction equal to $\mathrm{B}_{0}=0.134 \mathrm{~T}$. The magnetic field modulation frequency can vary from 1 to $200 \mathrm{~Hz}$. It has been proven experimentally that the optimal value is $f_{m}=50$ $\mathrm{Hz}$, since it provides a high value of the signal noise ratio and at the same time allows investigating a large number of media (Davydov et al., 2017c; Karseev et al., 2015). Reduction in the degree of inhomogeneity of the magnetic field was achieved by placing inserts 2 (Fig. 1) at the poles of the magnets in the form of steps (shims) of soft magnetic material (ARMCO iron). The weight of the magnetic system is $2.84 \mathrm{~kg}$; the size is $168 \times 132 \times 114 \mathrm{~mm}$. These parameters make it possible to consider our magnetic system as compact in comparison with magnetic systems of stationary NMR spectrometers and relaxometers.

To record NMR signals in a new design of a magnetic system using a modulation technique, a measuring probe was developed (Fig. 2). It consists of a registration coil 6 , a modulation coil 5 and a device for placing a container (cuvette) 7 (Fig. 1). The inner diameter of the cuvette 8 is $3.2 \mathrm{~mm}$, and the outer diameter is 4.8 $\mathrm{mm}$. In high-resolution NMR spectrometers, the cuvette diameters are 3.0 and $4.6 \mathrm{~mm}$, respectively.

\section{Fig. 2}

The measuring probe and the container for medium

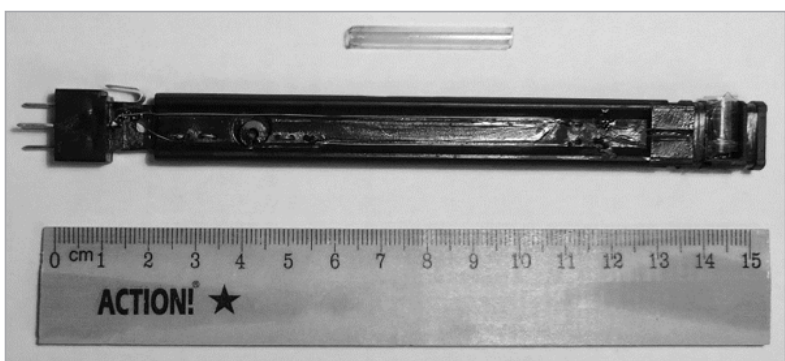


The investigated medium is placed in a cuvette and placed in a magnetic system 1 (Fig. 1), in which the NMR signal is detected. Fig. 3 shows, as an example, the detected NMR signals (the change in the voltage $U_{S}$ at the output of the autodyne detector 10 from the time) from water at the resonance frequency of the hydrogen nuclei (protons) $f_{H}=5705643 \mathrm{~Hz}$. The obtained result shows that it is impossible to measure $T_{1}$ and $T_{2}$ from the detected NMR signal (Fig. 3a) because of the large amount of noise. Therefore, an accumulation

Fig. 3

The detected NMR signal from water: a) without accumulation; b) at the output of the accumulation scheme

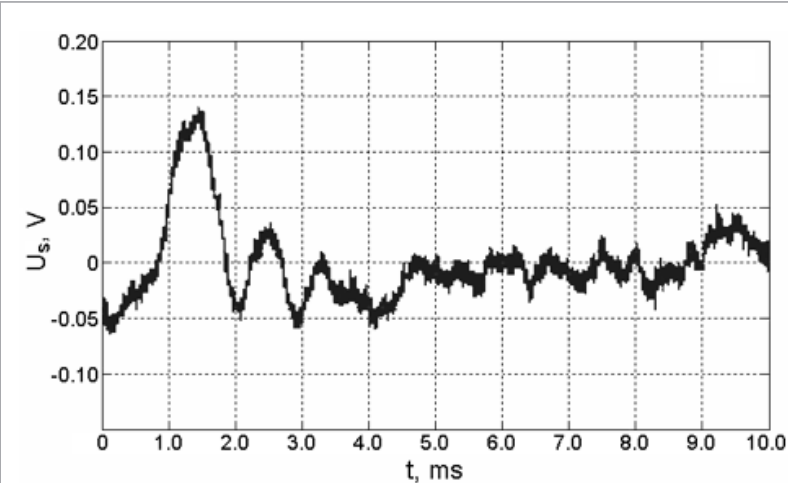

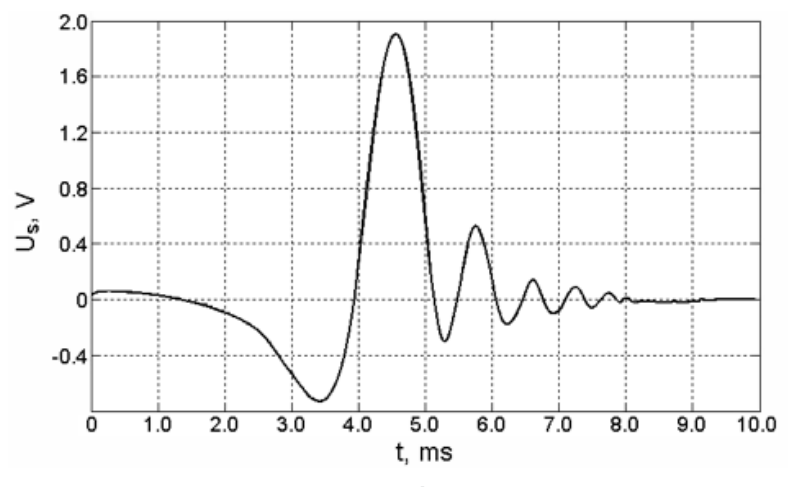

b scheme is used. After 128 accumulations (in time, it is less than $1 \mathrm{~s}$ ), the NMR signal acquires a stable line shape (Fig. 3b), allowing measurements.

At a water temperature of $\mathrm{T}=292.1 \mathrm{~K}$, using the detected NMR signal, we measured $\mathrm{T}_{1}=1.72 \pm 0.01 \mathrm{~s}$ and $T_{2}=3.12 \pm 0.03 \mathrm{~ms}$. The obtained values of $T_{1}$ and $T_{2}$ correspond to the standard state of water. This means that there are no impurities in it.
Fig. 4 shows, as an example, the detected NMR signals at the resonance frequency of hydrogen nuclei $f_{H}=5705643 \mathrm{~Hz}$ from water containing phosphorus. This chemical element is used in chemical fertilisers and pest control agents in agricultural fields. Phosphorus is often present both in soil and in various aquatic systems located near agricultural land.

\section{Fig. 4}

The detected NMR signal from water with phosphorus: a) without accumulation; b) at the output of the accumulation scheme
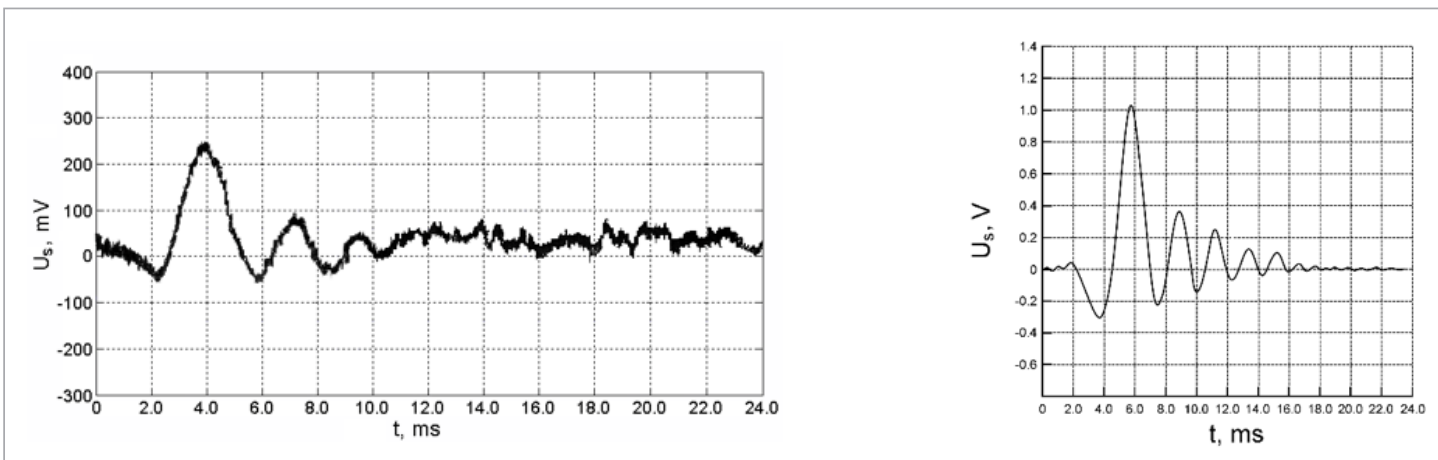
We measured $T_{1}=2.03 \pm 0.02 \mathrm{~s}$ and $T_{2}=9.18 \pm 0.09 \mathrm{~ms}$ for a given aqueous medium at a temperature of $T=292.1 \mathrm{~K}$. The measured values of $T_{1}$ and $T_{2}$ do not correspond to the values of the relaxation constants corresponding to the standard water state. Based on this result, it can be concluded that there are foreign substances or impurities in the water. Since the deviation in the values of $T_{1}$ and $T_{2}$ from the standard state is significant, this medium is dangerous. The substances that caused this deviation can be established only in a stationary laboratory; therefore, a sample of the medium must be delivered to it.

One of the experimental verifications of the operation of the developed compact NMR spectrometer was carried out on a tap water at $\mathrm{T}=18.9^{\circ} \mathrm{C}$. Using a compact NMR spectrometer, relaxation times of water were measured 10 times. Having this data, the average relaxation times and the measurement errors were calculated using standard techniques. The obtained values were $T_{1}=1.472 \pm 0.013 \mathrm{~s}$ and $\mathrm{T}_{2}=1.957 \pm 0.018 \mathrm{~ms}$. In addition, a sample of this water was studied on a stationary industrial NMR relaxometer Minispec mq 20 (developed by BRUKER). The obtained values were $T_{1}=1.4638 \pm 0.0044 \mathrm{~S}$ and $\mathrm{T}_{2}=1.9442 \pm 0.0058 \mathrm{~ms}$ (measurement error $0.003 \%$ ). The obtained values of relaxation times coincided within the measurement error.

This is a brief description of a methodology that was used to determine the measurement error. In other articles (Davydov et al., 2015; Myazin et al., 2018), this methodology is described in detail.

\section{Results and Discussion}

In the case of recording the spectrum (dependence of the NMR signal amplitude on the change in the frequency of the autodyne detector $f_{a}$ ) from the investigated medium, the situation with the determination of its state changes. Fig. 5 shows, as an example, the registered NMR spectrum from water with phosphorus at $\mathrm{T}=292.3 \mathrm{~K}$.

\section{Fig. 5}

Water NMR spectrum. Graph 1 corresponds to the NMR signal from phosphorus nuclei; graph 2 corresponds to the NMR signal from protons

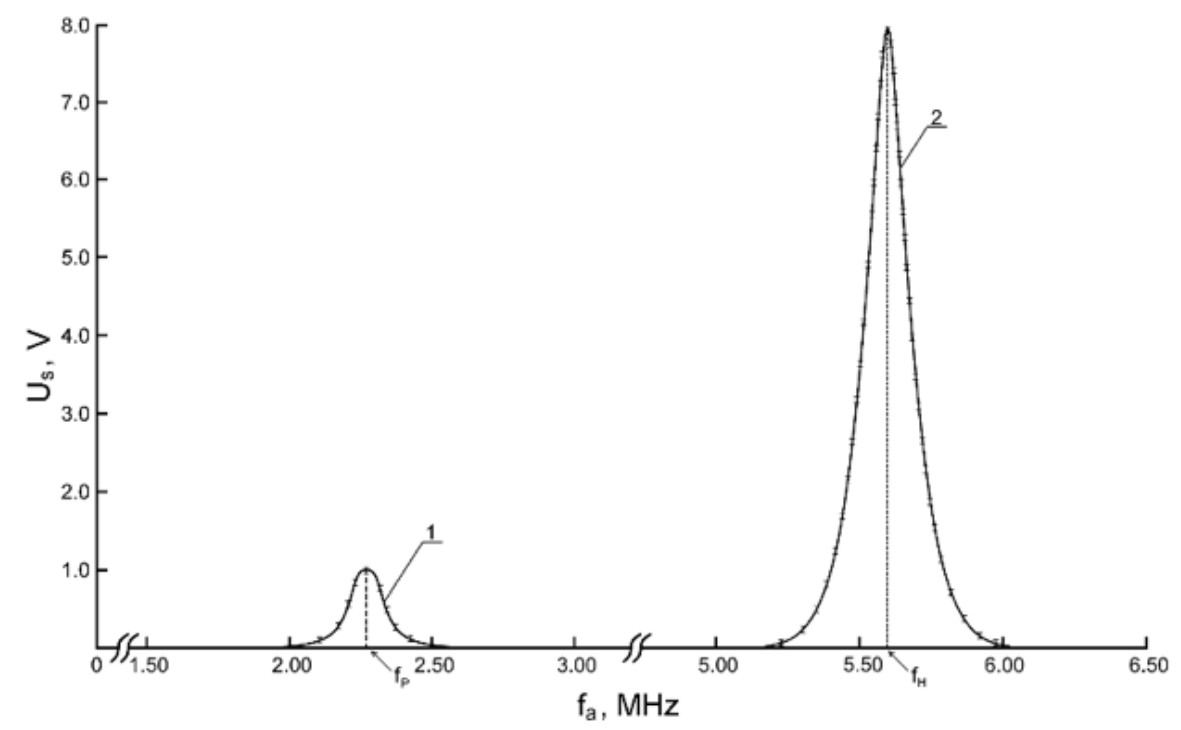

The obtained result shows the presence of dissolved phosphorus in water. The ratio of the amplitudes in the recorded NMR spectrum with allowance for the gain factors established that water contained $2 \%$ of phosphorus. The information obtained for the causes of the deviation in water, as well as the concentration 
of phosphorus in it, allows (without additional studies in a stationary laboratory) making a qualified decision about further use of this water (for example, irrigation of agricultural fields, technical purposes, cleaning, etc.).

Registration of the medium spectrum via an NMR signal also makes it possible to make the process of preparation of various fertilisers for their use more rational and reliable, in contrast to the case of control over the measured values of $T_{1}$ and $T_{2}$. Fig. 6 shows, as an example, the medium spectrum from an aqueous solution of potassium nitrate at $T=291.4 \mathrm{~K}$.

Potassium nitrate is actively used as a fertiliser to increase the yield of various plants. For its effective and safe use, it is necessary to control the potassium concentration, as well as the amount of potassium contained in the soil, etc. For example, when potassium nitrate is used with water, it is necessary to observe a certain proportion between them for different cases. The obtained result on the basis of the NMR spectrum recording shows that potassium content in potassium nitrate is $44 \%$. This corresponds to the standard, and the liquid medium prepared from potassium nitrate can be used for fertilising agricultural products. At a temperature $T=293.2 \mathrm{~K}$, according to the detected NMR signal, we measured $T_{1}=2.21 \pm 0.02 \mathrm{~s}$ and $\mathrm{T}_{2}=12.71 \pm 0.12 \mathrm{~ms}$ of a water solution of potassium nitrate. The obtained result shows the presence of a deviation in water from the standard state. If we use the method of the medium state control by relaxation constants, then additional studies are required in a stationary laboratory to determine the potassium content in it. The registration of the spectrum allows eliminating these additional financial and time costs.

\section{Fig. 6}

NMR spectrum of water solution of potassium nitrate. Graph 1 corresponds to the NMR signal from potassium nuclei, graph 2 corresponds to the NMR signal from protons

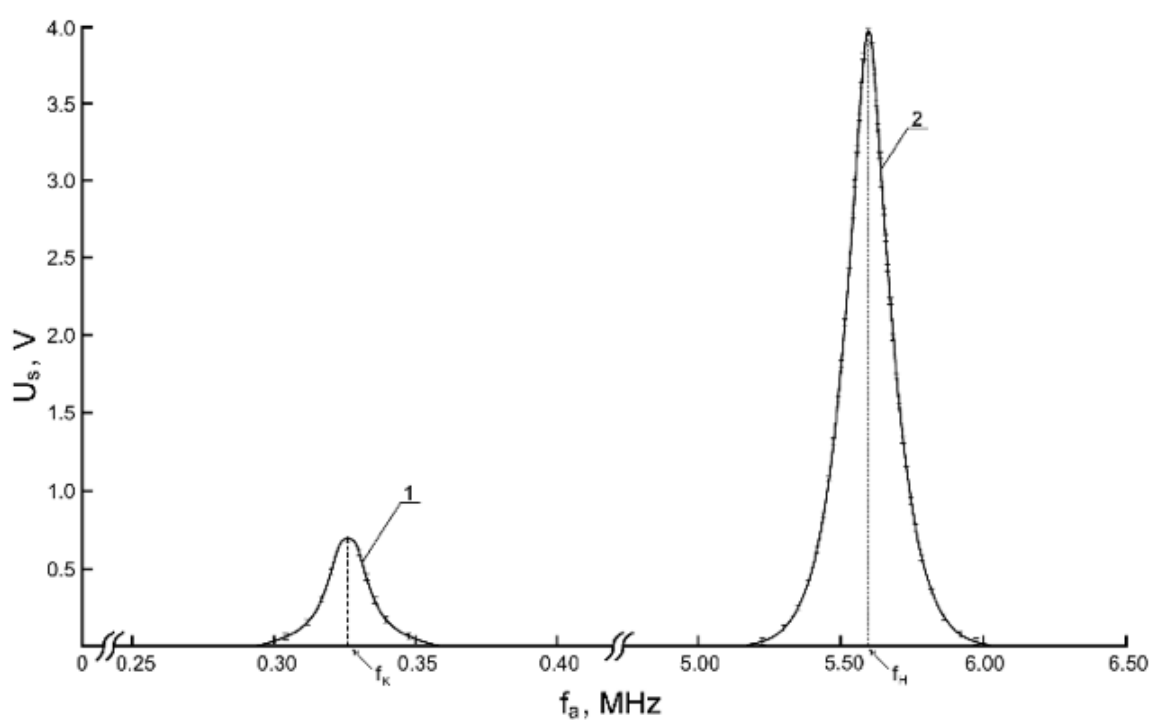

\section{Conclusions}

The use of the new developed design of a small-size NMR spectrometer in which advanced NMR signal detection techniques have been implemented has made it possible to significantly expand the possibilities for express control of the state of agricultural lands and reservoirs, as well as to more effectively solve other problems related to ecology.

If information about the medium has been lost, we can identify it by the signal from the nuclei with magnetic moments right at the site of the investigation, 
using information from the absorption spectrum registered in a weak magnetic field.

The use of the NMR method also makes it possible to check in the express mode various agricultural products (not only the liquid form). For example, verification of conformity with applicable standards can be carried out for various vegetables such as potatoes, beets, cucumbers, carrots, etc. This is possible since these vegetables contain chemical elements whose nuclei have magnetic moments.

\section{References}

Arkhipov, V.V. (2017) General purpose small Fourier spectrometers: Design and investigation. Instruments and Experimental Techniques 52: 692-695. https://doi.org/10.1134/ S0020441212050119

Bochenin V.I. (2007) Количественный рентгенофазовый экспресс-анализ сыпучих сырьевых материалов по регистрации дифракционного и характеристического излучения (Quantitative X-ray phase express analysis of rough bulk materials from the registration of diffraction and characteristic radiation). Дефектоскопия (Russian Journal of Nondestructive Testing) 11: 83-87. https://doi.org/10.1134/ S1061830907110095

Davydov V. V., Dudkin V. I., Karseev A. Yu. (2014) A compact nuclear magnetic relaxometer for the express monitoring of the state of liquid and viscous media. Measurement Techniques 8: 44-48. https://doi.org/10.1007/s11018-014-0559-0

Davydov V. V., Dudkin V. I., Myazin N. S., Rud' V. Yu. (2018) On the possibility of studying condensed media in the express mode using the nuclear-magnetic-resonance method. Instruments and Experimental Techniques 61: 140-147. https://doi. org/10.1134/S0020441218010220

Davydov V.V., Myazin N.S. (2017a) Compact multifunction nuclear-magnetic spectrometer. Measurement Techniques 2017. 2: 58 62. https://doi.org/10.1007/s11018-017-1171-x

Davydov V.V., Myazin N.S., Davydova T.I. (2017b) A nondestructive method for express testing of condensed media in ecological monitoring. Russian Journal of Nondestructive Testing 7: 52-61. https://doi.org/10.1134/S106183091707004X

Davydov V.V., Myazin N.S., Velichko E.N. (2017c) Characteristics of spectrum registration of condensed medium by the method of nuclear-magnetic resonance in a weak field. Technical Physics Letters 43: 607-610. https://doi.org/10.1134/ S1063785017070033

Davydov V.V., Velichko E.N., Dudkin V. I. and Karseev A. Yu. (2015) A nuclear magnetic relaxometer for express testing of the condensed medium conditions. Instruments and Experimental Techniques 58(2): 62-66. https://doi.org/10.1134/ S0020441215020062

Karseev A. Yu., Vologdin V.A., Davydov V.V. (2015) Features of nuclear magnetic resonance signals registration in weak magnetic fields for express - control of biological solutions and liquid medium by nuclear magnetic spectroscopy method. Journal of Physics: Conference Series 643: 012108. https://doi. org/10.1088/1742-6596/643/1/012108

Myazin N.S., Davydov V.V., Yushkova V.V., Davydova T.I., Rud' V.Yu. (2017) New nondestructive method for determining the composition of components in biological objects in express mode. Journal of Physics: Conference Series 917: 042017. https://doi.org/10.1088/1742-6596/917/4/042017

Myazin N.S., Davydov V.V., Yushkova V.V. and Rud V.Yu. (2018) On the possibility of recording absorption spectra in weak magnetic fields by the method of nuclear magnetic resonance. Journal of Physics: Conference Series 1038(1): 012088. https://doi. org/10.1088/1742-6596/1038/1/012088

Vitkovskii O. S., Marusina M. Y. (2016) Development of a standard system for metrological assurance of measurements of the pressure of saturated vapors of petroleum and petroleum products. Measurement Techniques 59: 247-251. https://doi. org/10.1007/s11018-016-0952-y 